\title{
Mucormycosis and COVID-19: A Narrative Review
}

\author{
Sharique Ahmad ${ }^{\text {* }}$, Shivani Singh ${ }^{1}$, Saeeda Wasim², Mohd. Anwar ${ }^{3}$, \\ Huma Parveen ${ }^{1}$ and Samarth Kaushikkumar Shah ${ }^{4}$
}

\author{
${ }^{1}$ Department of Pathology, Era's Lucknow Medical College and Hospital, Era University, \\ Lucknow, Uttar Pradesh-226003, India \\ ${ }^{2}$ Nova IVF Fertility, Hazratganj, Lucknow, U.P. - 226001, India \\ ${ }^{3}$ Department of Transfusion Medicine, SGPGI, Lucknow, Uttar Pradesh-226003, India \\ ${ }^{4}$ American University of Barbados, Wildey, Saint Michael, Barbados, BB11100
}

*Corresponding author

\section{A B S T R A C T}

\section{Keywords}

Mucormycosis, COVID-19,

Immunosuppressed, Diabetes mellitus

\section{Article Info}

Accepted:

20 June 2021

Available Online:

10 July 2021
Zygomycosis was identified as easy and comprehensive factor for 2 clinicopathological distinct infection, mucormycosis resulted due to Mucorales and entomophthoramycosis occur due to order Entomophthorales of class Zygomycota. The name Zygomycosis has been used as similar word only for mucormycosis. As mucormycosis is systemic fungal infection occurs in patients debilitated from metabolic or immune disorders. The changes in taxonomic classification and 3 of Zygomycota and disease Zygomycosis had been described. The Mucormycosis infection had been involved in patients with COVID-19 but its epidemics and diagnosis in patients are not exactly known. The cases of mucormycosis resulted due to post COVID-19 effect known till now helps in recognizing the risk factors, clinical features and outcome. It shows vulnerability towards the patients with comorbidities of diabetes mellitus mainly one with imperfect diabetes mellitus regulation and severe COVID-19 infection. Its presentation was severe in poorly regulated diabetes mellitus mainly rhino and cerebral orbital pathological symptoms. This fungal infection is fatal and occurs mainly in individuals with suppressed immune system due to diabetes mellitus, transplantation elevated serum level with iron and several other diseases which decrease our immune response had enhanced the risk of mucormycosis. The new treatment have to be strategizes urgently for understanding the pathogenesis of mucormycosis and response of host cell for penetrating the hyphae which will give the target for new therapeutic involvement. Currently the knowledge about the characters of the pathogen to multiply in host cell is the commonly known etiological factor for this fungal infection which Rhizopus oryzae. As the patients with higher level of serum and available iron are particularly prone towards the disease mucormycosis. This infection are extremely angioinvasive prominently involved with capability of organism for acquiring iron through host cell and interaction with endothelial cells of outer layer of blood vessels. Many strategies are in pre-clinical stage for identifying the exact diagnosis and prognosis of disease. 


\section{Introduction}

\section{Classification of the disease Mucormycosis}

The classification of kingdom fungi was established by Whittaker and the factor which leads to disease mucormycosis, entomophthoramycosis various other diseases from fungi produces aspetate hyphae and forms spore of either types zygospores or oospores classed under Phycomycetes of division Thallophyta (1). Nomenclature or we can say taxonomy of Fungi at that time was named due to their morphological homology of reproductive structure and three classes were known Basdiomycetes, Phycomycetes and Ascomycetes. The species which were known for reproducing asexually were classed under Deuteromycetes or imperfecti fungi (2). So understanding of biorhythm, nutrition mode and other aspects of organism elevate taxonomist attempt of placing organism in more nearly reflecting taxa on hypothetical evolutionary relationship. It results in adaptation of fungi to their own kingdom and undergoing remarkable alteration in classification. Phycomycetes contains gathering of non-related organism were classed in Phycomycetes was prohibited and their members were adjusted in classes of Zygomycetes, Chytridiomycetes, Hypochytridiomycetes, Trichomycetes, and Oomycete (3). Classification of fungi on shared derived characters basis demarcate the kingdom for inclusion of Chytridiomycota, Zygomycota, Ascomycota, and Basidiomycota and it had been working from decades before. The phylum Zygomycota consist Mucorales, Entomophthorales and other 8 order (4).

The modern taxonomy acknowledges the evolutionary association of organism showing their genomes. The taxonomist initiated molecular techniques for resolving fungal ancestors of all eukaryotes. The molecular phylogentic classification of fungi had been enhanced in past 15 years. Its analysis solved the Zygomycota ancestors sequence observation 3 nuclear ribosomal RNA subunits, RNA polymerase subunits, elongation factor $1 \mathrm{a}, 1 \mathrm{~b}$, protein tubulin, and small subunit of ribosomal DNA of mitochondria (5). This showed that Zygomycota consist various distinct monophyletic and also groups as entirely was paraphyletic or polyphyletic. Only one was present exceptionally RBP1 and 2 which showed Zygomycota as monophyletic (6). Though, investigation utilizing combination of various genes in inclusion of RNA polymerase subunit reveled it as polyphyletic phylum clearly (7).

The modification primarily attempted for Zygomycota was done through molecular phylogeny in 2001. Authors built phylogentic tree based on ribosomal RNA analysis and deleted arbuscular mycorrhizal from phylum Zygomycota and positioned it to new Glomeromycota phylum which was monophyletic. All the arbuscular mycorrhizal besides being obligate symbionts for vascular plants also forms clade with Zygomycota associated with other groups it had undergone further modifications in 2007.

This phylum undergoes further taxonomic changes by Hibbetin with collaboration of international taxonomist of this field had published a comparative classification of phylogentic type for kingdom fungi which was supported through monophyletic groups present evenly in various phylogentic analysis. The taxonomist utilized the data originated through molecular phylogentic analysis and also data found through multiple members of fungal taxonomy (8). The investigators provide a proposal for eliminating Zygomycoata due to the reason that phylum known polyphyletic and taxa positioned in Zygomycota conventionally and were assign in phylum Glomeromycota with four 
subphylum their broader relationship were undefined are named enigmatic taxa (9).

These groups relationships are not clearly known till now as reveled by investigators and many studies utilized sampling of taxon which were incomplete of Zygomycete ancestors (10). They also showed that Zygomycota could regenerate and verified for inclusion of Mucoromycotina and may additional studies in future lead to superior intensity of basal fungi group lineages. Consequently 6 gene phylogentic studies were utilized for reconstructing evolution in early phase of fungi which showed the consistent result in contrast of previous studies. So, altogether studies reveled Zygomycota as polyphyletic and other Mucoromycotina subphylum Entomopthoromycosis were diverged in two clades. Though in future studies of other clades could lead to enhanced resolution of lineages in fungi basal group.

The first incidence of this infection resulted through Mucorales member was published in 1885 by Paltauf. It was systemic infection seen in sinus and brain (rinocerebral), gastric which was described as Mycosis Mucorina by the authors (11). Though this shows that etiological factor observed more like Rhizopus species in contrast of Muco rowing to the sporangiospores which were unbranched and rhizoid-like structures. The fungal infection mucormycosis eventually being utilized by American pathologist for denoting mycosis resulted due to certain members of Mucorales (12).

The time before kingdom fungi was established the species giving rise to one big multinucleated cell termed as coenocytic hypae were classed in Phycomycetes phylum it indicates both types of production zygospore and oospores (13). Phycomycetes members were known to be pathogenic for humans its was named phycomycoses for giving an appropriate comprehensive term mycoses for this disease occurred due to any of the Phycomycetes species (14). This term became fruitful for disease mycoses in it the etiological agent were not cultured and known to be an unidentified Phycomycetes phylum species histopathological examination sections. As later term Phycomycosis become accepted widely as an appropriate name of disease disregards of diversification in its etiology (15). Clark subsidize the utilization of term mucormycosis for disease resulted due to species Mucorales for distinguishing it from subcutaneous phycomycosis due to fungi associated with Entomophthorales also name Entomophthroromycosis was proposed for subcutaneous phycomycosis (16). For supporting the taxonomic modifications Ajello et al., in 1976 gave suggestion for replacing zygomycosis name with mucormycosis (17).

This infectious disease named includes any mucosis resulted due to 2 orders Mucorales species with genera which includes Rhizopus, Absidia and Saksenaea and Cunninghamel also two other genera Basidiobolus and Conidiobolus. Since very long Zygomycosis name had been pronounced succeeding as synonym of mucormycosis (18) (19) (20).

Zygomycosis use instead of mucormycosis because Basidiobolusranarum (filamentous fungus) when seen during examination tissue section shows hyphaeof cross section along with eosinophilic material. Angioinvasion of pulmonary blood vessel mucormycosis obstructed by hyphae of Cunninghamella bertholletiae fungi which were stained with hematoxylin-eosin. It lacks eosinophillic material around hyphae taxonomy of fungi causing Mucormycosis are not commonly seen in case of this infection. As few of the fungal infection resulted due to the Entomophthorales though found not differentiable from classic mucormycosis. Contradicting to this mucormycosis known to 
consist disease occurred by any Mucorales member not through organism from Mucor genus (21). Few investigators revealed that mucormycosis should be named mucoralomycosis for denoting the order Mucorales not genus Mucor. Though, it is reasonable that order had been increased to Mucoromycotina which is subphylum and also it is surely possible that taxa could elevates to Mucoromycota as illustration of taxa enhances in this case the name mucoralomycosis be changed to mucoromycosis.

Anyway Mucorales or Zygomycota future fate in field of classification would resist revision of taxonomy. Though, entomophthoramycosis could imitate mucormycosis exceptionally till now number of systemic entomophthoramycosis cases known showed only bit of proportion among all zygomycosis incidence. The suitable name for this infectious disease must denoted typically inspite of exceptional cases and could provide stability of nomenclature that could not be disturbed by taxonomic alterations. Mucormycosis and entomophthoramycosis both of the name are known to be immersed in medical literature from several years and now replacement of zygomycosis from these two names are necessary as correct time had been reached.

Mucormycosis is any fungal infection causes through growing filamentous fungi belonging to class Glomeromycelin because current classification had replaced the name Zygomycetes and order Mucorales. Mostly the species in Mucor of genera Rhizopus, Absidia and Cunninghamella genra are to be incriminated (22). Commonly this infection occur from soil, buildings, damps etc it had been featured by hyphae arising in and surrounding of blood vessels and could be fatal for diabetic patients or critically low immunity due to any disease in individuals. Both of these terms Mucormycosis and
Zygomycosis are to be implied interchangeable, Zygomycota is added in modern fungus classification as it had been recognized as polyphyletic. It had been included in Zygomycosis includes Entomophthorales and eliminate Mucormucosis.

\section{Pathogenesis of mucormycosis}

This is a rare fungal disease of order Mucorales and after analysis of patients suffering with disease Rhizopus oryzae is commonly found in them responsible for $70 \%$ incidence of Mucormycosis (23) (24) (25). The most prominent factor of mucormycosis includes unregulated diabetes, ketoacids various forms of problems leading to increase acidity of body metabolic acidosis which requires treatment with corticosteroid, transplantation, trauma, low neutrophil counts and hematologic malignancies and deferoxamine treatment in patients with hematologic disorders. Due to elevated number of cases of diabetes, transplantation, cancer in US population the intensity of this fatal disease had increased drastically (26)(27). Unfortunately, inspite of disfiguring debridement and antifungal treatment the entire death rate for this disease is as usual $50 \%$ and its occurrence in infectious person with disseminated disease or with constant neutropenia (28). There is urgent need of new strategies for preventing and treating mucormycosis.

There are several evidences clearly demonstrating that individuals lacking cells involve in fighting against any pathogen like phagocytes or abnormalities in these cells are found to be at greater risk for disease mucormycosis likely to this low count of neutrophil also found to be involved in increasing the risk for this disease. In contrary to Acquired immunodeficiency syndrome (AIDS) patients are not found to be involved 
in enhancing the risk for developing this infection (29). It suggests that neutrophils but not T-lymphocytes are involved in inhibition of fungal spore proliferation. Though, phagocytes of both single nucleus and polymorphonuclear of normal host cells kills mucorales from the genesis of energy from carbohydrate by oxygen and cationic peptides function as effectors molecule against any pathogen associated defense (30). Currently performed studies revealed the neutrophils exposure to Rhizopus oryzae resulted in elevated regulation of Toll-like receptor 2 with strong proinflammtory expression of gene results immediate activation of NF-kB pathway it helps in proinflammtory response (31).The hyperglycemia condition and decreased $\mathrm{pH}$ present in patient of diabetes, ketoacidosis result in non-function phagocytes in them and diminished chemotaxis improper killing within the cell through oxidative and non- oxidative process. All these clinical findings and inhalation of Mucorales sporangiospores in suppressed immune response do not shown mucormycosis formation. Beside this corticosteroidimmunosuppressed or with diabetic ketoacidosis consisting individuals death is seen progressively in pulmonary and hematogenously spreading infection (32). Therefore capability of inhaled sporangiospores for germinating and forming hypae in host cell is important for forming this fungal infection. While macrophage of alveolar which helps in presenting the antigen to adaptive immune cells were isolated from immunosuppressed mice of lungs found to be associated in ingestion and hindrance from Rhizopus oryzae germination sporangiospores. These macrophages have restricted capability of killing in vitro to the organism, macrophages of immunosuppressed mice of pulmonary alveolar shows that they were not able to prevent the sporangiospores germination in intranasal infection. The mechanism from which the immune defense cell phagocytes are unregulated through diabetes mellitus, ketoacidosis and steroid are not exactly known till now it have to be determined. Though, Mucorales should possess specific virulence traits which helps the organism to utilize the particular condition of immunosuppression and biological disability observed in few of infectious person (33). As skin is the primary barrier against any type of pathogenic infections which provides defense against them same scenario occurs in cutaneous mucormycosis seen by elevated number of possibility for formation of mucormycosis in individuals with destruction of this protective hurdle for pathogens. Mucormycosis factors are found to be impotent in invading intact skin. Though patients of trauma, burn, skin damage and maceration of skin enabling the organism to invade in deep tissues. So this organism can be arise through these condition also from contaminated water, soil, contaminated surgical dressing, tapes nonsterile had been known to be involved in forming cutaneous mucormycosis at primary level (34). Furthermore, mucormycosis could even invade through direct access from tongue depressor in infants or through wooden applicators used for mixing drugs to the immunosuppressed patients (35).

All of these suggest the shifting of disease mucormycosis from community adapted to hospital acquired infection in suspected host. This infection is mainly featured by angioinvasion which leads to blood clot in blood vessels thrombosis and frequent necrosis of tissue. Shortage in oxygen supply to the tissue due diminishing blood supply to tissues which are infected could prevent leukocytes transport and antifungal factors at the site of location from where this infection start. The presence of masses tumor in blood vessels participates to the capability of organism to disperse hematogenously at their desired target organs. Accordingly disruption 
and penetration from endothelial cells of extracellular matrix at outer lining of blood vessels is a important step in pathogenic strategy of Rhizopus oryzae.

The angioinvasion participates in ability of organism to hematogenously disperse to their desired target. Therefore, disruption and invasiveness from endothelial cells of extracellular matrix proteins lining blood vessels are prone to be a crucial step in pathogenic procedure of $R$. oryzae. Consequently, knowing the mechanism of procedure happens could lead to perspective of preventing or treating disease mucormycosis. Previously done studies had provided R.oryzae could attach to extracellular matrix laminin and collagen protein (36).

The Rhizopus oryzae strains had been known for attaching to endothelial cells of umbilical vein in vitro and penetrate through these cells by inducing endocytosis process. It leads to damage in endothelial cells and protection of endocytosis abolishes the capability of organism causing endothelial damage of cells. Currently recognized receptor glucose regulated protein (GRP78) mediates entrance by destruction of these endothelial cells. It had been identified as cellular protein stimulates through starvation which leads to low glucose level. It is also member of HSP70 protein found in endoplasmic reticulum (ER) as this HSP70 protein is major chaperone protein associated in various cellular processes like proper folding, assembly, identifying incorrectly folded protein for proteasomal destruction, regulation of calcium level and function as sensor in ER. Instead of its function as chaperone protein various findings reported the GRP78 translocation to multiple cell surfaces. (37). The higher concentration of both glucose and iron reconcilable seen during diabetes and ketoacidosis increase the GRP78 expression on cells surface resulting invasion and destruction of endothelial cells from
Mucorales in receptor dependent manner. A study in a mice revealed that mice with diabetic ketoacidosis elevates vulnerability towards this disease and had enhanced GRP78 expression in brain, sinus, lungs in comparison to healthy mice also it should be noted that serum anti- GRP78 protected mice with diabetic ketoacidosis from fungal infection of mucormycosis (38). Though it had been not known whether immune serum with antiGRP78 could prevent anyone who have low counts of neutrophils due to mucormycosis. So all these findings provide novel perception of specific vulnerability of patients with diabetic ketoacidosis to infection mucormycosis and could give a new ideas for therapeutic interventions. As many other fungi found to be non-pathogenic like Aspergillus fumigates in Drosophila melanogaster which is a species of fly, Mucorales frequently infects and destroy it by killing the wild type of flies. The entire genome expression profiling of normal wild type flies infected from $R$. orzyae were compared with A. fumigates revealed down regulated genes by $R$. oryzae which are involved in recognition of pathogens, defensive immune system, detoxification, stress response, tissue repair, metabolism regulating steroid.

The Zygomycetes class contains aseptate hyaline moulds which could perform both sexual and asexual reproduction. As this fungus is present ubiquitously in soil and decaying vegetation. Among all 5 genera order mucorales are involved for causing disease in humans. There is not any susceptibility based on age or sex. Most of the cases were of acute surgery emergencies through few chronic forms had been seen with developing symptoms in 4 week of period. Initial location of penetration are seen in sinuses, lungs, skin and Gastro-intestinal tract. The symptoms signs pathological findings are known to be identical in mucormycosis nevertheless of its etiology. This fungi shows 
preference for lymph vessels invasion, resulting extensive blood clot and necrosis of tissue present nearly and lymphatic penetration could occur at later stage of infection. The high blood glucose and acidic environment present in patients with ketoacidotic diabetes mellitus favors Rhizopus growth.

\section{COVID-19 and Mucormycosis}

The COVID-19 is known for disease in entire world. While various treatment options are evaluated for it but none of them beside systemic glucocorticoids had been known for improving survival in COVID-19. Unfortunately, use of glucocorticoids in abundance could lead to secondary fungal or bacterial infections. Invasive pulmonary aspergillosis (IPA) entangling the course of COVID-19 recognized on larger scale. Therefore, mucormycosis is diagnosed or suspected uncommonly, glucocorticoids are not very expensive and are available widely and shown decreased in mortality in hypoxemic patients with COVID-19 (39). However, it could elevate the use of synchronous immunomodulatory drugs like a tocilizumab which could increase the risk of infections in COVID-19 patients (40). Pulmonary mucormycosis are increasingly diagnosed when fatality has improved overtime, successful management of mucormycosis could be performed by controlling hyperglycemia treatment at early stage with amphotericin B and surgery. However, COVID-19 had made a specific scenario where all components of management are compromised, primarily hyperglycemia is aggravated by most effective treatment for severe patients of COVID-19 namely glucocorticoids. Together with acute respiratory distress syndrome and multiple organ failure preventing timely testing and diagnostic imaging (41). One of the observations seems was conventional risk factors like diabetes mellitus, hematological malignancies, transplantation. The formation of mucormycosis could probably be attributed to the utilization of glucocorticoids and suggests a requirement for judicious use. Though, use of glucocorticoids in severe cases of COVID-19 or utilization of glucocorticoids in higher doses should be ignored. The absence of drugs advantage and its targets to immune pathways should not be utilized like tocilizumab (42). Although, physicians containing critically ill COVID-19 patients should be aware from serious infections which could complicate the course of COVID-19. An elevated intensity of clinical suspicion is required for pulmonary mucormycosis diagnosis. Early diagnosis and timely management are essential for improving the outcomes of pulmonary mucormycosis.

As this disease had effected more than millions people in entire world. Although aspergillosis a fungal infection have been reported for complicating moderated patients of COVID-19 known as COVID-19associated aspergillosis (CAPA), physiological process involved in disease and real incidence are to be discussed most of these incidence are to be documented from biopsy. Additionally, Mucorales infections raised as a major concern in COVID-19 as with uncontrolled diabetes mellitus and other disease are vulnerable for mucormycosis infection and moderate COVID-19 patients also utilizing corticosteroid for treating these critical patients is the known factor for mucormycosis.

In addition to hyperglycemia and abnormalities of metabolism associated with iron had been seen in moderate COVID-19 cases. The severity of this infection with iron associated abnormalities can be named hyperferritinemia syndrome but the elevated level of ferritin could be a marker for moderate systemic disease is not clear as 
against of pathophysiological modulator is also not known till now. Inspite of their role elevated level of ferritin could lead its increment intracellularly which forms reactive oxygen species and further tissue damage.

The signaling molecules responsible for immune regulation cytokines mainly IL-6 and diabetic ketoacidosis because of moderate COVID-19 infection stimulates ferritin synthesis and down regulates iron export leading its intracellular encumber which aggravate the mechanism (43). The damage of tissue results to free iron release into circulation aggravate of iron noticed is one of the key and particular risk factor of mucormycosis. Though other known explanation for mucormycosis and COVID-19 is endothelialtis seen in moderate patients. Series of autopsy reports revealed that moderate pulmonary vascular endothelial damage and angiogenesis in patients collapsed from this disease than who are died from influenza A. Other reports shown that endothelial injured patients collapsed due to multiple organ damage. The early step of mucormycosis is invasion from endothelial adhesion. Hyperglycemia and acidic condition induces receptor GRP78 present on endothelial cell and Mucorales adhesion spore coat proteins homologs from accurate storm for enhancing invasion and attachment of Mucorales to endothelium. Interestingly, GRP78 is known receptors for gaining entry and leading to COVID-19 infection (44).

Mucormycosis had appeared for intersecting two crises one COVID-19 and other is imperfect diabetes mellitus in this pandemic. A sharp contrast for COVID-19-associated aspergillosis and most of COVID-19associated mucormycosis incidence are known. Clinicians must have a greater awareness and concern of critical illness with keeping mucormycosis and COVID-19 association differential in critically ill patients and diabetes mellitus particularly if rhinoorbital or cerebral presentation had been noticed. The COVID-19 along with diabetes mellitus consisting patient's experiences orofacial pain without swelling which can be a neurological pain it is autonomous and without facial asymmetry sudden cell death necrosis these findings do not revealed any kind of indication for Mucormycosis cases (45). Though prevention for antifungal treatment had been unproven, high level of blood sugar control is found to be one of the prominent components for prevention and imaging of disease mucormycosis.

Though mucormycosis is an rare infection to be found in healthy individual but various immunosuppressed condition predispose it which include diabetes mellitus with or without diabetic ketoacidosis, malignances, low neutrophil counts for long time, transplantation medication of corticosteroid, hemochromatosis, immunodeficiency syndrome, lack of nutrition, open wound. This fungal disease includes nose, joints, heart, kidney, gastrointestinal tract (GIT), sinuses, central nervous system (CNS), mediastinum but Rhino-orbital-cerebral-mucormycosis (RCOM) is most common variety observed in clinical practice in entire world. Though it should be observed that ROCM denotes to whole spectrum ranged from restricted sinonasal disease progression to orbits which further leads to orbital-cerebral disease. The involvement of place could depend on condition like RCOM is more frequently seen in uncontrolled diabetes and diabetes ketoacidosis on other hand pulmonary association had also be seen in patients with low count of neutrophil, organ transplant, hematological malignancies and GIT results in malnourished individuals. The pathological hallmarks for mucormycosis are giant cell invasion, eosinophilic necrosis and thrombosis. Microbiological observation shows hyphae identification based on size of 
septa clinical diagnosis performed by Smith and Krichner (1950) gave a standard it had been considered as standard method till now which includes black, necrotic turbinates's, crabby blood, blood with red or brown discharge and pain in facial portion of both same sides and swelling in soft portion around eyes or nose with discoloration and blepharoptosis and ophthalmoplegia with various cranial nerve palsies not associated with documented defects. A study conducted in 2019 showed 388 confirmed or suspected mucormycosis cases in India preceding to this disease of COVID-19 revealed 18\% of patients were consisting diabetes ketoacidosis and $57 \%$ of them were having uncontrolled diabetes mellitus (46). Similarly, 465 mucormycosis cases not containing COVID19 infection had revealed rhinoorbital presentation which was seen commonly followed by cutaneous and pulmonary types. The persuading factors involved in mucormycosis in India consist diabetes mellitus, transplantation, and malignancy(47).

Currently a systemic review organized which reported 41 mucormycosis cases in COVID19 infected individuals further diabetes mellitus was seen in 93\% cases and 88\% attained corticosteroid (48). Altogether, all of this shows impious trinity for mucormycosis diabetes and corticosteroid in patients of COVID-19. As till now not a single study have been compared for mucormycosis infected individual without diabetes COVID19 infection and not received steroid in comparison of patients received steroid and formed mucormycosis. So, it is not easy to establish association between COVID-19 and mucormycosis in context of corticosteroid intake. The presence of high blood sugar and diabetic ketoacidosis precipitation isseen because of consumption of corticosteroids. Beside the use of corticosteroids reduction in immune defensive cells and phagocytic function of WBC results to broncho-alveolar macrophages improper functioning, ingestion and fusion endolysosome enhances the vulnerability of diabetic patient of forming mucormycosis. As this infection results to endothelialitis, lymphopenia and decreased level of cell surface marker CD4+ and CD8+ and predisposes to secondary or adaptable infection of this fungus, availability of iron in unbound form is a prominent resource for mucormycosis infection. As hyperglycemia leads to glycosylation of protein transferrin helps in iron transport and ferritin further decreases iron binding and permits elevated unbound iron. Elevation of cytokines in COVID-19 patient with interlukin-6 enhances the unbound iron which increases ferritin amount due to enhanced synthesis and reduced iron transport. Further accompanying acidosis elevates free iron by same process and additionally by reduction in capability of transferrin to chelate iron, hyperglycemia, decreased $\mathrm{pH}$, unbound iron and ketenes in existence of decrease phagocytosis activity of WBC increases the mucor growth. Additionally expression GRP-78 of endothelium and fungal ligand spore covering homolog protein enables angioinvasion, metastases and necrosis (49). It is possible that mucormycosis cases reported could be underrepresented for actual responsibility of owing the hurdles in diagnosis histopathological or microbiological mainly in the outbreak of disease. While few of the cases reported consist not enough details and either do not reported fruitful parameter. Ultimately, it is difficult to evaluate the consequences in individual with mucormycosis and infection of COVID-19 at this time due to these data and reports had been published and various patients are currently under diagnosis. As mucormycosis is an opportunistic systemic fungal infection which is rare but vulnerably found in immunosuppressed people due to COVID-19 and various co-morbidities which are treated through corticosteroids. Mainly till now the 
findings and reports have revealed that patients of COVID-19 with diabetes mellitus, organ transplantation and various diseases leading to suppress immune system are prone towards the disease mucormycosis. Conclusively, it could be possibly that clinicians should observe for evaluation of mucormycosis in COVID-19 infection. Further research is needed for evaluating potential link between these two infections elevation in mucormycosis. It had been seen that unholy intersection of diabetes, use of corticosteroid and COVID-19, beside these three cytokine storm, endothelial damage, lymphopenia. Optimal hypoglycemia and evidence based on usage of corticosteroid in COVID-19 infected individual had been suggested for reducing the burden of fatal mucormycosis. The extensive analysis main virulence agent like utilization of $R$. oryzae develop plans that translate the intervening clinical trials. It requires time effort for study design analysis and implementation. The advantages of interventions complementing existing therapies could be profound in patients with mucormycosis. All together this reveled that for avoiding the disease due to COVID-19 associated treatment involving corticosteroid. The treatment should be performed with appropriate strategy of both advantageous and disadvantageous of therapy. There is urgent requirement for properly understanding and treating mucormycosis association with COVID-19 disease.

\section{References}

1. Whittaker R H. New concepts of kingdoms of organisms. Evolutionary relations are better represented by new classifications than by the traditional two kingdoms. Science 1969; 163:150-60.

2. Emmons C W, Binford C H, Utz J P, KwonChung K J. Medical mycology. 3rd ed. Philadelphia: Lea \& Febiger, 1977:254-84.

3. Dyer, Owen. Covid-19: India sees record deaths as "black fungus" spreads fear.
2021373:1238; BMJ Clinical research.

4. Grossman M E, Fox, Lindly P Kovarik C, Rosenbach M. Subcutaneous and deep mycoses: Zygomucosis/ Mucormycosis. Cutaneous Manifestations of Infection in the Immunocompromised Host (2nd ed.) Springer. 2012; 51-58.

5. James T Y, Kauff F, Schoch C L, et al., Reconstructing the early evolution of fungi using a six-gene phylogeny. Nature 2006; 443:818-22.

6. Liu Y J, Hodson M C, Hall B D. Loss of the flagellum happened only once in the fungal lineage: phylogenetic structure of kingdom Fungi inferred from RNA polymerase II subunit genes. BMC EvolBiol 2006; 6:7486.

7. Keeling $\mathrm{P}$ J. Congruent evidence from alpha-tubulin and beta-tubulin gene phylogenies for a zygomycete origin of Microsporidia. Fungal Genet Biol 2003; 38:298-309.

8. Hibbett D S, Binder M, Bischoff J F, et al., A higher-level phylogenetic classification of the Fungi. Mycol Res 2007; 111:509-47.

9. Moreau F. Les champignons: physiologie, morphologie, de'veloppmentetsyste' matique. Vol 2. Paris: Lechevalier, 1954.

10. Steenkamp E T, Wright J, Baldauf S L. The protistan origins of animals and fungi. MolBiolEvol 2006; 23:93-106.

11. Paltauf A. Mycosis mucorina: einBeitragzurKenntnis der menschilchenFadenpiltzer-krankungen. Virchows Arch PatholAnat 1885; 102:54364.

12. Baker R D. Pulmonary mucormycosis. Am J Pathol 1956; 32:287-313

13. Clements F E, Shear C L. The genera of fungi. New York: The H. W. Wilson Co, 1931.

14. Kian Joe L, Njo-Injo T E, Pohan A, Van der Muelen $\mathrm{H}$, Emmons $\mathrm{C} \quad \mathrm{W}$. Basidiobolusranarum as a cause of subcutaneous phycomycosis in Indonesia. AMA Arch Dermatol 1956; 74:378-83.

15. Kian Joe L, Njo-Injo T E, Tjokronegro S, Schaafma S J, Emmons C W. Phycomycosis 
of the central nervous system associated with diabetes mellitus in Indonesia. Am J Clin Path 1959; 32:62-70.

16. Clark B M. The epidemiology of phycomycosis. In: Wolstenholme GEW, Porter R, eds. Systemic mycoses. London: J \& A Churchill, 1968:179-205.

17. Ajello L, Dean D F, Irwin R S. The zygomycete Saksenaeavasi form is as a pathogen of humans with a critical review of the etiology of zygomycosis. Mycologia 1976; 68:52-62

18. Kasai M, Harrington S M, Francesconi A, et al., Detection of a molecular biomarker for zygomycetes by quantitative PCR assays of plasma, bronchoalveolar lavage, and lung tissue in a rabbit model of experimental pulmonary zygomycosis. J ClinMicrobiol 2008; 46:3690-702.

19. Schwarz P, Bretagne S, Gantier J C, et al., Molecular identification of zygomycetes from culture and experimentally infected tissues. J ClinMicrobiol 2006; 44:340-9.

20. Hata D J, Buckwalter S P, Pritt BS, Roberts G D, Wengenack N L. Real-time PCR method for detection of zygomycetes. J ClinMicrobiol 2008; 46:2353-8.

21. Baker R D. Mucormycosis, a new disease? JAMA 1957; 163:805-8

22. Rippon J. Medical Mycology. Philadelphia, PA: WB Saunders. 982:615-637.

23. Ribes J A, Vanover-Sams C L, Baker D J. Zygomycetes in human disease. ClinMicrobiol Rev 2000; 13:236-301.

24. Spellberg B, Edwards J Jr, Ibrahim A. Novel perspectives on mucormycosis: pathophysiology, presentation, and management. ClinMicrobiol Rev 2005; 18:556-69.

25. Roden M M, Zaoutis T E, Buchanan W L, et al., Epidemiology and outcome of zygomycosis: a review of 929 reported cases. Clin Infect Dis 2005; 41:634-53.

26. Ibrahim A S, Edwards J E, Filler S G. Zygomycosis. In: Dismukes WE, Pappas PG, Sobel JD, eds. Clinical mycology. New York, NY: Oxford University Press, 2003: 241-51. 7.

27. Marr K A, Carter R A, Crippa F, Wald A,
Corey L. Epidemiology and outcome of mould infections in hematopoietic stem cell transplant recipients. Clin Infect Dis 2002; 34:909-17.

28. Gleissner B, Schilling A, Anagnostopolous I, Siehl I, Thiel E. Improved outcome of zygomycosis in patients with hematological diseases? Leuk Lymphoma 2004; 45:135160.

29. Sugar A M. Agents of mucormycosis and related species. In: Mandell GL, Bennett JE, Dolin R, eds. Principles and practice of infectious diseases. 6th ed. Philadelphia, PA: Elsevier, 2005: 2979.

30. Sugar A M. Agents of mucormycosis and related species. In: Mandell GL, Bennett JE, Dolin R, eds. Principles and practice of infectious diseases. 6th ed. Philadelphia, PA: Elsevier, 2005: 2979

31. Chamilos G, Lewis R E, Lamaris G, Walsh T J, Kontoyiannis D P. Zygomycetes hyphae trigger an early, robust proinflammatory response in human polymorphonuclear neutrophils through toll-like receptor 2 induction but display relative resistance to oxidative damage. Antimicrob Agents Chemother 2008; 52:722-4.

32. Lamaris G A, Ben-Ami R, Lewis R E, Chamilos G, Samonis G, Kontoyiannis D P. Increased virulence of Zygomycetes organisms following exposure to voriconazole: a study involving fly and murine models of zygomycosis. J Infect Dis 2009; 199:1399-406.

33. Kwon-Chung $\mathrm{K} \quad \mathrm{J}$, Bennett $\mathrm{J}$ E. Mucormycosis. In: Medical mycology. Philadelphia, PA: Lea \& Febiger, 1992: 524-59.

34. Gartenberg G, Bottone E J, Keusch G T, Weitzman I. Hospital-acquired mucormycosis (Rhizopus rhizopodiformis) of skin and subcutaneous tissue: epidemiology, mycology and treatment. New Engl J Med 1978; 299:1115-8

35. Mitchell S J, Gray J, Morgan M E, Hocking M D, Durbin G M. Nosocomial infection with Rhizopus microsporus in preterm infants: association with wooden tongue depressors (see comments). Lancet 1996; 
348:441-3.

36. Bouchara J P, Oumeziane N A, Lissitzky J C, Larcher G, Tronchin G, Chabasse D. Attachment of spores of the human pathogenic fungus Rhizopus oryzae to extracellular matrix components. Eur J Cell Biol 1996; 70:76-83

37. Wang M, Wey S, Zhang Y, Ye R, Lee A S. Role of the unfolded protein response regulator GRP78/BiP in development, cancer, and neurological disorders. Antioxid Redox Signal 2009; 11:2307-16

38. Liu M, Spellberg B, Phan Q T, et al., The endothelial cell receptor GRP78 is required for mucormycosis pathogenesis in diabetic mice. J Clin Invest 2010; 120:1914-2.

39. Garg D, Muthu V, Sehgal I S, Ramachandran R, Kaur H, Bhalla A, et al., Coronavirus Disease (Covid-19) Associated Mucormycosis (CAM): Case Report and Systematic Review of Literature. Mycopathologia 2021, 1-10

40. Lionakis, M. S.; Kontoyiannis, D. P Glucocorticoids and invasive fungal infections. Lancet 2003, 362, 1828-1838.

41. Singh, A K, Gupta R, Ghosh A, Misra A. Diabetes in COVID-19: Prevalence, pathophysiology, prognosis and practical considerations. Diabetes Metab. Syndr. 2020: 14: 303-310.

42. Donnelly J P, Chen S C, Kauffman C A, Steinbach W J, Baddley J W, Verweij PE, et al., Revision and Update of the Consensus Definitions of Invasive Fungal Disease From the European Organization for Research and Treatment of Cancer and the Mycoses Study Group Education and
Research Consortium. Clin. Infect. Dis. 2020: 71: 1367-1376.

43. Lamoth $F$, Lewis $R$ E, Walsh $T J$, Kontoyiannis D P. Navigating the uncertainties of COVID-19 associated aspergillosis (CAPA): A comparison with influenza associated aspergillosis (IAPA). J. Infect. Dis. 2021.

44. Perricone C, Bartoloni E, Bursi R, Cafaro G, Guidelli G M, Shoenfeld Y, et al., COVID19 as part of the hyperferritinemic syndromes: The role of iron depletion therapy. Immunol. Res. 2020, 68, 213-224

45. Sabirli R, Koseler A, Goren T, Turkcuer I, Kurt O. High GRP78 levels in Covid-19 infection: A case-control study. Life Sci. 2021, 265, 118781

46. Mishra N, Mutya VSS, Thomas A, et al., A case series of invasive mucormycosis in patients with COVID-19 infection. Int $\mathbf{J}$ Otorhinolaryngol Head Neck Surg. 2021 May;7(5):867-87'

47. Prakash H, Ghosh A K, Rudramurthy S M, et al., A Prospective Multicenter Study on Mucormycosis in India: Epidemiology, Diagnosis, and Treatment. Med. Mycol. 2019;57:395-402.

48. John T M, Jacob C N, Kontoyiannis D P. When Uncontrolled Diabetes Mellitus and Severe COVID-19 Converge: The Perfect Storm for Mucormycosis. J Fungi (Basel). 2021 Apr 15;7(4):298.

49. Baldin C, Ibrahim A S. Molecular mechanisms of mucormycosis -The bitter and the sweet. PLoSPathog 2017;13(8): e1006408.

\section{How to cite this article:}

Sharique Ahmad, Shivani Singh, Saeeda Wasim, Mohd. Anwar, Huma Parveen and Samarth Kaushik Kumar Shah. 2021. Mucormycosis and COVID-19: A Narrative Review. Int.J.Curr.Microbiol.App.Sci. 10(07): 598-609. doi: https://doi.org/10.20546/ijcmas.2021.1007.065 